\title{
Local Defect Correction Techniques Applied to a Combustion Problem
}

\author{
Martijn Anthonissen \\ Technische Universiteit Eindhoven, Scientific Computing Group \\ m.j.h.anthonissen@tue.nl
}

Summary. The standard local defect correction (LDC) method has been extended to include multilevel adaptive gridding, domain decomposition, and regridding. The domain decomposition algorithm provides a natural route for parallelization by employing many small tensor-product grids, rather than a single large unstructured grid. The algorithm is applied to a laminar Bunsen flame with one-step chemistry.

\section{Introduction}

Partial differential equations (PDEs) with solutions that have highly localized properties appear in many application areas, e.g. combustion, shock hydrodynamics, and transport in porous media. Such problems require a fine grid only in the region(s) of high activity, whereas elsewhere a coarser grid suffices. We consider a discretization method for elliptic boundary value problems introduced by Hackbusch [1984]. In this technique, the local defect correction (LDC) method, the discretization on the composite grid is based on a combination of standard discretizations on uniform grids with different spacings that cover different parts of the domain. The coarse grid must cover the entire domain, and its spacing is chosen in agreement with the relatively smooth behavior of the solution outside the high activity areas. Apart from this global coarse grid, one or more local fine grids are used that are also uniform, each of which covers only a (small) part of the domain and contains a high activity region. The grid spacings of the local grids are chosen in agreement with the behavior of the continuous solution in that part of the domain.

The LDC method is closely related to the fast adaptive composite grid (FAC) method (McCormick [1984a], McCormick and Thomas [1986]). An important difference with LDC is that an explicit discretization scheme for the composite grid is proposed, in which special difference stars near the grid interfaces are used. The resulting discrete system is solved by an iterative method which may take advantage of the composite grid structure. This is a crucial difference with the LDC method, which combines standard discretizations on uniform grids only and does not use an a priori given composite grid dis- 
cretization. For the FAC method in a variational setting, convergence results have been given by McCormick [1984b]. The variational theory is extended to the finite volume element method in McCormick and Rüde [1994]. Xu [1992] presents an abstract framework and general convergence theory for a wide range of iterative methods, among which domain decomposition, multigrid and multilevel methods. He groups the algorithms in parallel and successive subspace correction methods. Xu shows in particular that FAC is equivalent to classic multigrid with smoothing in the area of refinement only.

The idea to approximate low frequency components on a coarse grid and high frequency components on a (local) fine grid forms the basis of multigrid. McCormick and Ruge [1986] present unigrid, an algorithm based on these principles and especially suited for testing the feasibility of using multigrid in a given application. Xu and Zhou $[1999,2000,2001]$ use the fact that the global behavior is dominated by low frequencies and the local behavior by high frequencies to design discretization schemes in a finite element context. They study elliptic boundary value problems and prove error estimates for the finite element solution. Based on these estimates, they develop several algorithms. The simplest one is to solve a global problem on a locally refined grid. This algorithm is improved by using a residual correction technique, in which a global coarse grid problem is solved first. Next the coarse grid residual is corrected by solving the problem on one or more locally refined grids that cover the whole domain but are very coarse outside the area of refinement. Note that the global coarse grid problem needs to be solved only once and is not coupled with the local problems. This is different from the LDC method, in which the local problems take artificial boundary conditions from the coarse grid solution and cover part of the domain only. Xu and Zhou $[1999,2000]$ present parallel versions of their algorithms by subdividing the domain in disjoint subdomains. In Xu and Zhou [2000], further algorithms are developed by ignoring the lower order terms of the PDE on the local grids, which can be done because the symmetric positive definite part dominates the high frequency components. Xu and Zhou [2001] study a solution technique for nonlinear elliptic PDEs. The full nonlinear problem is first discretized by a standard finite element technique on a global coarse grid. Next, the residual is corrected using linearized discretizations on fine grids.

This paper deals with some extensions to the standard LDC method; we add adaptivity, multilevel refinement, domain decomposition and regridding. We apply the new algorithm to a Bunsen flame problem previously treated by Bennett and Smooke [1998].

\section{Formulation of the LDC method}

Before presenting our extensions to the LDC method, we begin by describing the standard LDC method. We consider the elliptic boundary value problem

$$
\left\{\begin{aligned}
L u=f, & & \text { in } \Omega, \\
u=g, & & \text { on } \partial \Omega .
\end{aligned}\right.
$$


In (1), $L$ is a linear elliptic differential operator, and $f$ and $g$ are the source term and Dirichlet boundary condition, respectively. Other types of boundary conditions can be used as well, but for ease of presentation we formulate the method for (1). To discretize (1), we first choose a global coarse grid (grid spacing $H$ ), which we denote by $\Omega^{H}$. An initial approximation $u_{0}^{H}$ on $\Omega^{H}$ can be found by solving the system

$$
L^{H} u_{0}^{H}=f^{H},
$$

which is a discretization of (1). In (2), the right-hand side $f^{H}$ incorporates the source term $f$ as well as the Dirichlet boundary condition $g$. We assume $L^{H}$ to be invertible.

Assume that the continuous solution $u$ of (1) has a high activity region in some (small) part of the domain. We select a subdomain $\Omega_{l} \subset \Omega$ such that the high activity region of $u$ is contained in $\Omega_{l}$. In $\Omega_{l}$, we choose a local fine grid (grid spacing $h$ ), which we denote by $\Omega_{l}^{h}$, such that grid points of the global coarse grid that lie in the area of refinement also belong to the local fine grid. In order to formulate a discrete problem on $\Omega_{l}^{h}$, we define artificial boundary conditions on $\Gamma$, the interface between $\Omega_{l}$ and $\Omega \backslash \Omega_{l}$. We apply an interpolation operator $P^{h, H}$ that maps function values at coarse grid points on the interface to function values at fine grid points on the interface. In the numerical simulations we use linear interpolation. In this way, we find the following approximation $u_{l, i}^{h}$, iteration $i=0$, on $\Omega_{l}^{h}$ :

$$
L_{l}^{h} u_{l, i}^{h}=f_{l}^{h}-B_{l, \Gamma}^{h} P^{h, H}\left(\left.u_{i}^{H}\right|_{\Gamma}\right) .
$$

In (3), matrix $L_{l}^{h}$ (assumed to be invertible) is a discrete approximation to $L$ on the subdomain $\Omega_{l}$. The first term on the right-hand side incorporates the source term $f$ as well as the Dirichlet boundary condition $g$ on $\partial \Omega_{l} \backslash \Gamma$ given in (1). In the second term, the operator $B_{l, \Gamma}^{h}$ represents the dependence of the fine grid points on the coarse grid solution at the artificial boundary $\Gamma$.

We will now use the local fine grid solution to update the coarse grid approximation. If we were able to substitute the projection on $\Omega^{H}$ of the exact solution $u$ of boundary value problem (1) into the coarse grid discretization (2), we would find the local discretization error or local defect $d^{H}$, given by $L^{H}\left(\left.u\right|_{\Omega^{H}}\right)=f^{H}+d^{H}$. We could then use $d^{H}$ within the right-hand side of (2) to find a better approximation on the coarse grid. However, as we do not know $u$, we instead use the fine grid approximation $u_{l, 0}^{h}$ to estimate $d^{H}$ at the coarse grid points inside the area of refinement $(x, y) \in \Omega_{l}^{H}:=\Omega^{H} \cap \Omega_{l}$. We define $w_{0}^{H}$ as the global coarse grid function of best approximations so far:

$$
w_{0}^{H}(x, y):=\left\{\begin{array}{l}
u_{l, 0}^{h}(x, y),(x, y) \in \Omega_{l}^{H}, \\
u_{0}^{H}(x, y),(x, y) \in \Omega^{H} \backslash \Omega_{l}^{H},
\end{array}\right.
$$

and estimate the defect by $d^{H}=L^{H}\left(\left.u\right|_{\Omega^{H}}\right)-f^{H} \approx L^{H} w_{0}^{H}-f^{H}=: d_{0}^{H}$. Assuming that the stencil at grid point $(x, y)$ involves (at most) function 
values at $(x+i H, y+j H)$ with $i, j \in\{-1,0,1\}, d_{0}^{H}$ provides an estimate of the local discretization error of the coarse grid discretization at all points of $\Omega_{l}^{H}$. We apply the coarse grid correction step to find $u_{i}^{H}, i=1$ :

$$
L^{H} u_{i+1}^{H}= \begin{cases}f^{H}(x, y)+d_{i}^{H}(x, y), & (x, y) \in \Omega_{l}^{H}, \\ f^{H}(x, y), & (x, y) \in \Omega^{H} \backslash \Omega_{l}^{H} .\end{cases}
$$

Because (4) incorporates estimates of the local discretization error of the coarse grid discretization, $u_{1}^{H}$ is assumed to be more accurate than $u_{0}^{H}$. Hence it provides a better boundary condition on $\Gamma$, and a better solution on the local fine grid can be found by solving (3) with $i=1$. This leads to an iterative method: we can solve a new updated coarse grid problem.

Often one or two LDC iterations will suffice to obtain a satisfactory approximation on the composite grid due to the high rate of convergence of the method. Typically, iteration errors are reduced by a factor of 10 to 1,000 in each iteration step (cf. Ferket and Reusken [1996], Hackbusch [1984], Nefedov and Mattheij [2002], Anthonissen [2001]). A detailed analysis of the convergence behavior for diffusion equations is given in Anthonissen et al. [2003b].

\section{Extensions to the LDC method}

We now extend the LDC algorithm by adding adaptivity, multilevel refinement, domain decomposition, and regridding. The result will be a technique for discretizing and solving (1) on a composite grid found by adaptive grid refinement, given a code for solving boundary value problem (1) on a tensorproduct grid in a rectangular domain.

\section{Adaptive multilevel refinement}

We assume that the continuous solution $u$ has one area of high activity; it is straightforward to generalize the algorithm to the case where there is more than one area of high activity. We assume that the initial coarse grid is given by its $x$ - and $y$-coordinates $x_{i}, y_{j}$, and define the boxes $B_{i j}$ formed by grid points and points on the boundary, viz. $B_{i j}=\left(x_{i}, x_{i+1}\right) \times\left(y_{j}, y_{j+1}\right)$. In order to determine which boxes require refinement, we introduce the positive weight function of Bennett and Smooke [1998, 1999] as an indicator for solution roughness. As detailed in Anthonissen et al. [2003a], the weight function assigns a value to each box $B_{i j}$ and points will be added in regions where the weight function is large, so it should measure the rapidity of change of $u$. $\mathrm{Xu}$ and Zhou [2000] give theoretical justification why we may use a global a posteriori error estimate for equi-distribution of the error, as we do here. Apart from high activity boxes, we also flag their neighbors for refinement in order to prevent the solution from being artificially trapped at interfaces between coarse and fine grids, which can happen if high activity areas move during recalculation on the finer grid.

For the area of refinement $\Omega_{l}$, we choose the smallest rectangle that encloses all flagged boxes; more efficient choices are discussed in the next section. 
In $\Omega_{l}$, we choose a local fine grid $\Omega_{l}^{h}$ by uniform refinement. The integer refinement factor $\sigma$ is typically set to 2. Ideally, $\sigma$ should be chosen largest at places where the weight function is largest. However, this approach would lead to an unstructured composite grid, which we want to avoid. Therefore, we will use multiple refinement levels.

After adding successive levels of refinement, the fine grid approximations are used to improve the coarse grid approximations via coarse grid correction steps. Once we have returned to the base grid, we will solve discrete problems on finer levels again.

\section{Domain decomposition}

In the previous section, we determined the smallest rectangle enclosing all flagged boxes and chose to refine this rectangle entirely. However, this approach may refine many boxes that have not been flagged for refinement, especially when an area of high activity is not aligned with the grid directions. To remedy this inefficiency as well as to prevent the grids from becoming too large, we combine the multilevel LDC algorithm with domain decomposition, in which we use a set of rectangles to cover all flagged boxes. We require each flagged box to be enclosed in at least one rectangle, and we want the rectangles to be overlapping. The overlap of the rectangles is necessary in situations where interfaces between rectangles intersect high activity zones. We remedy large errors at these interfaces by performing a number of domain decomposition iterations via a standard multiplicative Schwarz procedure.

To find a set of rectangles satisfying the conditions just stated, a cost function is defined that states how expensive using a certain set is. The algorithm evaluates the cost of using a single rectangle (as we did in the previous section), splitting it horizontally in two smaller rectangles or splitting vertically. This procedure is performed recursively on the smaller rectangles if splitting has occurred; see Anthonissen et al. [2003a] for details. The algorithm, including the Schwarz alternating procedure, is shown in Figure 1.

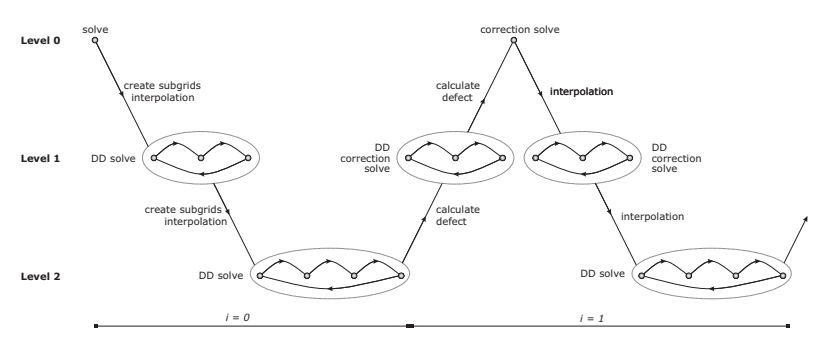

Fig. 1. Solution procedure with domain decomposition.

\section{Regridding}

Refining a grid and solving the boundary value problem on the new composite 
grid may cause the region(s) of high solution activity to move. Therefore, we apply the regridding procedure from Bennett and Smooke [1998] before proceeding from Level $l$ to Level $l+1$.

\section{Application to a combustion problem}

We now turn our attention to the axisymmetric laminar Bunsen flame with one-step chemistry. This problem was previously presented by Bennett and Smooke [1998]. Because almost all of the dependent variables in the Bunsen flame problem have large gradients in a very small region of the computational domain, adaptive gridding is a must for this simulation. The physical configuration for the Bunsen flame is shown in Figure 2. A mixture of methane and air flows up from a central jet, which is surrounded by a coflowing air stream. A steady conical flame forms at the mouth of the cylindrical burner.

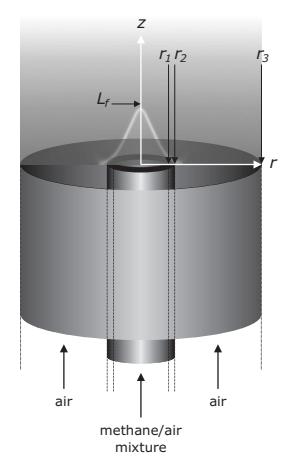

Fig. 2. Physical configuration for the axisymmetric Bunsen flame.

The chemical model we consider has five species: methane, oxygen, water, carbon dioxide, and the abundant inert, nitrogen. There are nine dependent variables in the Bunsen flame problem: radial velocity, axial velocity, vorticity, temperature, and five mass fractions. These variables satisfy a set of strongly coupled nonlinear PDEs, see Anthonissen et al. [2003a] for details. The initial coarse grid is chosen to be more finely spaced in the region above the inner jet, because it is known that the flame forms in that area. The exact $r$ - and $z$-coordinates of the initial grid are given in Anthonissen et al. [2003a].

Due to the nature of the LDC method, the PDEs need only be discretized on tensor-product grids. We apply standard finite difference stencils at interior points. First-order upwinding is used on convective terms. Details can be found in Anthonissen et al. [2003a]. The discretized governing equations and boundary conditions form a system of equations, that is linearized by a damped, modified Newton's method (Deuflhard [1974], Smooke [1983]) with a nested Bi-CGSTAB linear algebra solver; the latter is preconditioned using a block Gauss-Seidel preconditioner. 


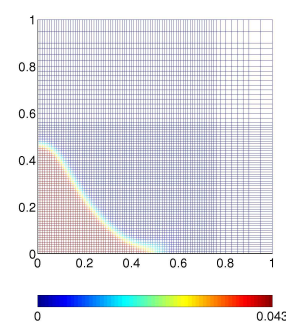

(a) $l_{\max }=0$.

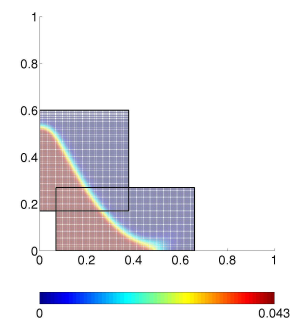

(b) $l_{\max }=1$.

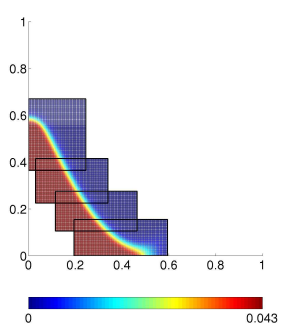

(c) $l_{\max }=2$.

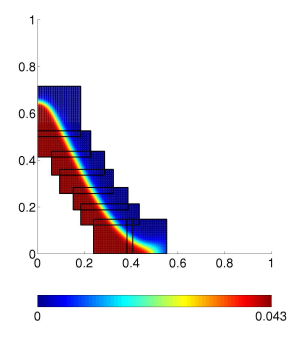

(d) $l_{\max }=3$.

Fig. 3. Plots of the methane mass fraction on the finest level for the LDC simulations with various values of the maximum level of refinement $l_{\max }$.

Four different LDC simulations have been carried out on a $175 \mathrm{MHz}$ SGI Octane with 1 GB of RAM. Each simulation starts from an already converged solution on the base tensor-product grid. For each first solve on a local fine grid, we use as an initial guess the approximation found by interpolating the approximation on the parent grid. At each level with more than one grid, five domain decomposition iterations are done to improve the boundary conditions at the internal interfaces. The flagging of high activity boxes is based on the methane mass fraction. The refinement factor is 2 .

Figure 3 shows the projection of the methane mass fraction on LDC composite grids with increasingly fine resolution. Although the flame structure is similar in each plot, the flame length increases, with the largest increase occurring when the first refinement level is added. In Anthonissen et al. [2003a], the LDC results are shown to have excellent agreement with both the local rectangular refinement (LRR) results for the same problem as well as with results found on equivalent tensor-product (ETP) grids with the same resolution presented by Bennett and Smooke [1998]. In the LRR method, an unstructured grid is constructed from an initial tensor-product grid by flagging and refining high activity boxes individually. Unlike tensor-product grids, grid lines in an LRR grid are not required to extend from one domain boundary to the other. A Newton solver is subsequently applied to the discretized PDE system on the complete unstructured grid.

In the LDC simulations, however, the Newton solver is applied to many small grids individually rather than to one large grid. In the LDC simulation for $l_{\max }=3$, the composite grid consists of the initial tensor-product grid with three additional refinement levels, that have two, four, and eight subgrids, respectively. The biggest tensor-product grid in this hierarchy has only 16,653 points - a substantial memory savings over the ETP grid of 312,872 points.

\section{Conclusions}

In this paper, we have extended the standard LDC method by including multilevel adaptive gridding, domain decomposition, and regridding. We have successfully applied this method to a lean axisymmetric laminar Bunsen flame 
with one-step chemistry. In the future, we would like to investigate the inclusion of more sophisticated domain decomposition techniques within the method, so that fewer iterations will be required among grids at a given level. We would also be interested in the possibility to solve different problems on the global coarse and local fine grid, using e.g. chemical equilibrium; $c f . \mathrm{Xu}$ and Zhou [2000, 2001] in which only the symmetric positive definite part or a linearized discretization is solved locally. As there are virtually no conceptual hurdles in expanding the approach to higher dimensions, our ultimate goal is to apply the extended LDC method to three-dimensional combustion problems, for which its low memory usage and parallelization opportunities will play an important role.

\section{References}

M. J. H. Anthonissen. Local defect correction techniques: analysis and application to combustion. PhD thesis, Eindhoven University of Technology, Eindhoven, 2001.

M. J. H. Anthonissen, B. A. V. Bennett, and M. D. Smooke. An adaptive multilevel local defect correction technique with application to combustion. Technical Report RANA 03-24, Eindhoven University of Technology, Eindhoven, Oct. 2003a.

M. J. H. Anthonissen, R. M. M. Mattheij, and J. H. M. ten Thije Boonkkamp. Convergence analysis of the local defect correction method for diffusion equations. Numerische Mathematik, 95(3):401-425, 2003b.

B. A. V. Bennett and M. D. Smooke. Local rectangular refinement with application to axisymmetric laminar flames. Combust. Theory Modelling, 2:221-258, 1998.

B. A. V. Bennett and M. D. Smooke. Local rectangular refinement with application to nonreacting and reacting fluid flow problems. J. Comput. Phys., 151:684-727, 1999.

P. Deuflhard. A modified Newton method for the solution of ill-conditioned systems of nonlinear equations with application to muliple shooting. Num. Math., 22:289-315, 1974.

P. J. J. Ferket and A. A. Reusken. Further analysis of the local defect correction method. Computing, 56:117-139, 1996.

W. Hackbusch. Local defect correction and domain decomposition techniques. In K. Böhmer and H. J. Stetter, editors, Defect Correction Methods. Theory and Applications, Computing, Suppl. 5, pages 89-113, Wien, New York, 1984. Springer.
Suls.

S. McCormick. Fast adaptive composite grid (FAC) methods. In K. Böhmer and H. J. Stetter, editors, Defect Correction Methods: Theory and Applications, pages 115-121. Computing Supplementum 5, Springer-Verlag, Wien, 1984a.

S. McCormick. Fast adaptive composite grid (FAC) methods. In K. Böhmer and H. J. Stetter, editors, Defect Correction Methods: Theory and Applications, pages 115-121. Computing Supplementum 5, Springer-Verlag, Wien, 1984b.

S. McCormick and U. Rüde. A finite volume convergence theory for the fast adaptive composite grid methods. Appl. Numer. Math., 14:91-103, 1994.

S. McCormick and J. Ruge. Unigrid for multigrid simulation. Math. Comp., 41:43-62, 1986.

S. McCormick and J. Thomas. The fast adaptive composite grid (FAC) method for elliptic equations. Math. Comp., 46(174):439-456, 1986.

V. Nefedov and R. M. M. Mattheij. Local defect correction with different grid types. Numerical Methods for Partial Differential Equations, 18:454-468, 2002.

M. D. Smooke. Error estimate for the modified Newton method with applications to the solution of nonlinear, two-point boundary value problems. J. Optim. Theory Appl., 39:489-511, 1983.

J. Xu. Iterative methods by space decomposition and subspace correction. SIAM Review, 34(4): 581-613, December 1992.

J. Xu and A. Zhou. Some local and parallel properties of finite element discretizations. In C.-H. Lai, P. E. Bjørstad, M. Cross, and O. B. Widlund, editors, Eleventh International Conference on Domain Decomposition Methods, pages 140-147, Bergen, 1999. Domain Decomposition Press.

J. Xu and A. Zhou. Local and parallel finite element algorithms based on two-grid discretizations. Mathematics of Computation, 69(231):881-909, 2000.

J. Xu and A. Zhou. Local and parallel finite element algorithms based on two-grid discretizations for nonlinear problems. Advances in Computational Mathematics, 14(4):293-327, 2001. 